\title{
Impacto da saúde bucal na qualidade de vida de adolescentes grávidas
}

\author{
Impact of oral health on the quality of life of pregnant adolescents
}

\section{Impacto de la salud bucal en la calidad de vida de las adolescentes embarazadas}

\author{
Daniela Pereira LIMA ${ }^{1}$ \\ Suzely Adas Saliba MOIMAZ ${ }^{2}$ \\ Cléa Adas Saliba GARBIN ${ }^{2}$ \\ Maria Lúcia Marçal Mazza SUNDEFELD ${ }^{3}$ \\ Tânia Adas SALIBA ${ }^{3}$
}
${ }^{1}$ Mestre e Doutora em Odontologia Preventiva e Social, Programa de Pós-Graduação em Odontologia Preventiva e Social, Faculdade de Odontologia de Araçatuba, UNESP-Univ Estadual Paulista, Araçatuba - SP, Brasil ${ }^{2}$ Professora Titular do Programa de Pós-Graduação em Odontologia Preventiva e Social, Faculdade de Odontologia de Araçatuba, UNESP-Univ Estadual Paulista, Araçatuba - SP, Brasil ${ }^{3}$ Professora Adjunto do Programa de Pós-Graduação em Odontologia Preventiva e Social, Faculdade de Odontologia de Araçatuba, UNESP-Univ Estadual Paulista, Araçatuba-SP, Brasil

\begin{abstract}
Resumo
Objetivo: avaliar o impacto da saúde bucal na qualidade de vida de adolescentes grávidas e identificar os fatores sóciodemográficos que podem influenciar nesse conceito. Material e método: estudo transversal tipo inquérito e levantamento das condições de saúde bucal de 127 adolescentes grávidas. Para mensurar o impacto da saúde bucal na qualidade de vida foi utilizado o OHIP-14, aplicado com um questionário sócio-demográfico. As condições de saúde bucal foram analisadas, empregando-se os Índices CPO-D, SiC-Index, e IPC de acordo com os critérios estabelecidos pela OMS. Foram realizadas análises estatísticas descritivas, e o teste Exato de Fisher com nível de significância de 5\%. Resultados: Não houve adolescentes nas classes sociais A1 e A2. A grande maioria 79,5\% relatou impacto das condições bucais em sua qualidade de vida. $\mathrm{O}$ item que demonstrou maior impacto foi a dor física, manifestada por 23,6\% das participantes. O índice CPO-D foi 12,51 ( $\mathrm{dp}=4,21)$ e o $\mathrm{SiC}$ Index foi de 18,85. O percentual de livres de cárie foi 6,3\% e 91,3\% apresentavam problemas periodontais. Houve associação estatística significante entre as variáveis: impacto na qualidade de vida e doença periodontal $(\mathrm{p}=0,0311)$. Conclusão: Por se tratar de um estudo realizado em Unidades Básicas de Saúde não houve gestantes nos níveis sociais mais elevados. As adolescentes apresentaram alto índice de cárie dentária e doença periodontal, revelando saúde bucal deficiente. A maioria relatou impacto na qualidade de vida em decorrência de problemas bucais, sendo a presença de "dor física" a maior responsável pelas repercussões negativas na vida destas meninas.
\end{abstract}

Descritores: Saúde Bucal; Qualidade de Vida; Adolescente; Gravidez.

\begin{abstract}
Objective: To evaluate the impact of oral health on the quality of life of pregnant adolescents and to identify the sociodemographic factors that may influence this concept. Material and methods: Transversal study, survey type and survey of the oral health status of 127 pregnant adolescents. To measure the impact of oral health on the quality of life, the OHIP-14 was used and applied with a socio-demographic questionnaire. The oral health statuses were analyzed, using the DMFT, SiC-Index and CPI indices according to the criteria established by the WHO. Descriptive statistical analysis, and the Fisher's exact test with a significance level of 5\% were performed. Results: There were no adolescents in the A1 and A2 social classes. The majority, 79.5\% reported the impact of oral conditions on the quality of life. The item that showed the greatest impact was physical pain, manifested by $23.6 \%$ of the participants. The DMFT index was $12.51(\mathrm{SD}=4.21)$ and the $\mathrm{SiC}$ Index was 18.85 . The percentage of caries-free was $6.3 \%$, and $91.3 \%$ had periodontal problems. There was a significant statistical association between the variables: impact on the quality of life and periodontal disease $(p=0.0311)$. Conclusion: Because it is a study in Basic Health Units, no pregnant women were in the higher social levels. The adolescents had high rates of dental caries and periodontal disease, revealing poor oral health. Most reported impact on the quality of life due to oral health problems, and the presence of "physical pain" was most responsible for the negative impact on the lives of these girls. Descriptors: Oral Health; Quality of Life; Adolescent; Pregnancy.
\end{abstract}

\section{Resumen}

Objetivo: Evaluar el impacto de la salud bucal en la calidad de vida de las adolescentes embarazadas y para identificar los factores sociodemográficos que pueden influir en este concepto. Métodos: Estudio transversal tipo de estudio y encuesta del estado de salud oral de 127 adolescentes embarazadas. Para medir el impacto de la salud bucal en la calidad de vida se utilizó OHIP-14, aplicado con un cuestionario sociodemográfico. Se analizaron las condiciones de salud oral, utilizando los índices de CPOD, SiC Índice IPC y de acuerdo con los criterios establecidos por la OMS. Se realizaron análisis estadístico descriptivo, y la prueba exacta de Fisher con un nivel de significación del 5\%. Resultados: No hubo adolescentes de las clases A1 y A2. La mayoría del 79,5\% reportado impacto de las condiciones orales en la calidad de vida. El artículo que mostró el mayor impacto fue el dolor físico expresada por el 23,6\% de los participantes. El índice CPOD fue 12,51 (SD = 4,21) y el Índice de SiC fue 18.85. El porcentaje de libres de caries fue del 6,3\% y el 91,3\% tienen problemas periodontales. Hubo una asociación estadística significativa entre las variables: impacto en la calidad de vida y la enfermedad periodontal $(\mathrm{p}=0,0311)$. Conclusión: Debido a que es un estudio en unidades básicas de salud había mujeres embarazadas en los niveles sociales más altos. Los adolescentes tenían altos índices de caries dental y la enfermedad periodontal, dejando al descubierto la mala salud oral. La mayoría reportó impacto en la calidad de vida debido a problemas dentales y la presencia de "dolor físico" más responsable por el impacto negativo en la vida de estas chicas.

Descriptores: Salud Bucal; Calidad de Vida; Adolescente; Embarazo.

\section{INTRODUÇÃO}

A adolescência compreende o período da vida que vai dos 10 aos 19 anos de idade e sucede a infância. ${ }^{1}$ Tem início com a puberdade, e se caracteriza por uma série de mudanças corporais, psicológicas, fisiológicas e sociais ${ }^{2}$.
Segundo o censo de 2010, o Brasil registra 190.755.799 milhões de habitantes, sendo que $17,9 \%$ estão no período da adolescência, estimando-se que a população feminina seja de 17 milhões $^{3}$. 
Esse período caracteriza-se por ser uma fase crítica na vida da adolescente, pois a jovem se apresenta bastante vulnerável aos riscos relativos à saúde, de modo que seu comportamento pode oscilar por momentos exacerbados de atitudes positivas referentes à autoestima e cuidados com a aparência, e momentos de atitudes negativas, quando se tornam negligentes com seus cuidados à saúde ${ }^{4,5}$.

A gestação nessa faixa etária vem sendo considerada, em alguns países, problema de saúde pública, uma vez que pode acarretar complicações obstétricas, com repercussões para a mãe e o recém-nascido, bem como problemas psicossociais e econômicos ${ }^{6}$.

No mundo, aproximadamente $25 \%$ das mulheres têm seu primeiro filho antes dos 20 anos de idade, podendo se observar taxas ainda maiores em países em desenvolvimento. No Brasil um terço da população total é constituída por jovens a baixo de 20 anos de idade, respondendo por um milhão de gravidezes/ano ${ }^{3}$.

Em relação à saúde bucal, embora a adolescência seja reconhecida por um período no qual os jovens podem apresentar risco aumentado à cárie dentária e à doença periodontal, em virtude do precário controle de placa bacteriana e redução dos cuidados com a higiene bucal ${ }^{7}$, associado a gravidez, esse comportamento pode trazer vários prejuízos não só a mãe, mas também a seus bebês, isto porque a ocorrência de doença periodontal, durante o período gestacional pode estar associado à pré-eclâmpsia e constitui um fator de risco significante para o nascimento de crianças prematuras de baixo peso ao nascer além de ser um fator de risco para doenças cardiovasculares, diabetes e infecções respiratórias $8,9,10$.

Sabe-se que para a saúde bucal, tem sido um desafio desenvolver maneiras de avaliar saúde, visto que os indicadores tradicionalmente utilizados constituem medidas clínicas úteis para mensurar a necessidade normativa de tratamento, contudo não trazem informações a respeito do impacto que a condição bucal possui na qualidade de vida dos indivíduos ${ }^{11}$. Com o propósito de complementar esses indicadores, Slade e Spencer ${ }^{12}$ desenvolveram e testaram o indicador subjetivo Oral Health Impact Profile (OHIP) para avaliar o impacto social da doença bucal na vida das pessoas.

O OHIP foi originalmente desenvolvido, utilizando-se do modelo conceitual adaptado por Locker ${ }^{13}$ que relaciona a qualidade de vida com a saúde bucal e apresenta sete dimensões conceituais: limitação funcional, dor física, desconforto psicológico, incapacidade física, incapacidade psicológica, incapacidade social e deficiência. Desenvolvido originalmente no idioma inglês e em diferente contexto sociocultural, ao longo do tempo, foi traduzido em diversos idiomas ${ }^{14}$, inclusive traduzido transculturalmente e validado para o idioma português do Brasil ${ }^{15}$. Atualmente tem sido o instrumento mais utilizado para avaliar o impacto causado por condições bucais no bem-estar e na qualidade de vida das pessoas ${ }^{14}$. Ressalta-se ainda que as percepções, o conhecimento e o comportamento de adolescentes em relação à saúde bucal têm sido tema de estudo de diversos

autores do mundo, todavia, poucos estudos tratam do impacto da saúde bucal na qualidade de vida de adolescentes grávidas, representando uma lacuna importante ${ }^{14,15}$. Deste modo, o objetivo nesse estudo foi avaliar o impacto da saúde bucal na qualidade de vida de adolescentes grávidas e identificar os fatores sociodemográficos que podem influenciar diretamente nesse conceito.

\section{MATERIAL E MÉTODO}

Foi realizado um estudo transversal, tipo inquérito e levantamento das condições de saúde bucal de adolescentes grávidas. O universo populacional considerado para este estudo foram adolescentes gestantes que estavam em acompanhamento pré-natal no período de janeiro a maio de 2013, em 13 Unidades Básicas de Saúde do município de Araçatuba.

A cidade de Araçatuba pertence ao estado de São Paulo, Brasil, com 181.618 habitantes. A renda per capita é de cerca de US\$7,355.00 / ano, a taxa de mortalidade infantil é 10.29/100, e o Índice de Desenvolvimento Humano é 0,848. Desde 1972, foi adicionado 0,6 a 0,8 mg / 1 de flúor à água de abastecimento público municipal.

Para o cálculo amostral considerou-se a probabilidade convencional de $50 \%$ das pacientes apresentarem bolsa periodontal, desta forma, aceitando-se uma diferença entre a amostra e população de $10 \%$, o número mínimo de gestantes que participariam da amostra seria 96. Entretanto, para garantir as possíveis perdas de informações, foram acrescentados $25 \%$ a esse total, sendo o número mínimo estimado para a amostra deste estudo de 120 gestantes.

No decorrer do levantamento de dados em todas as Unidades Básicas de Saúde foi considerada a presença das gestantes um evento aleatório, uma vez que o pesquisador não sabia quem ou quantas adolescentes estariam presentes no momento da coleta dos dados, todavia, nossa amostra foi composta de 127 gestantes, número maior do que o mínimo planejado.

Foram incluídas na amostra adolescentes de 10 a 19 anos que estavam em atendimento pré-natal nas UBS de Araçatuba-SP que consentiram, após leitura e assinatura do Termo de Consentimento Livre e Esclarecido (TCLE). Pais ou responsáveis consentiram no caso das menores de idade.

Foi desenvolvido questionário sobre o perfil socioeconômico (classe social, renda, escolaridade) e questões que contemplam a gestação e o acesso aos serviços odontológicos e o motivo da ultima consulta odontológica. Para determinação da classe social, foi utilizado o Critério Padrão Brasil 2008, proposto pela Associação Brasileira de Empresas de Pesquisa (ABEP). ${ }^{16}$ Essa classificação leva em conta a posse de bens móveis e anos de estudo do chefe da família. Foram descritas oito categorias (A1, A2, B1, B2, C1, C2, D e E), em ordem decrescente, ou seja, a classe A1 é considerada a mais elevada e a $\mathrm{E}$ a mais desprovida;

Uma versão simplificada do Oral Health Impact Profile, o OHIP-14, conforme preconizado por Slade $(1997)^{17}$ foi utilizada para a avaliação do impacto da saúde bucal na qualidade de vida das adolescentes. Para calcular o impacto da saúde bucal na qualidade de vida, utilizou-se o chamado "método aditivo"; no qual as 14 respostas foram codificadas segundo uma escala de 5 pontos, variando de 0 para "nunca" até 4 para "sempre", e todos os valores atribuídos às respostas foram somados. Tais pontuações poderiam variar de 0 a 56 . Quanto maior a pontuação, maior a percepção do impacto da saúde bucal pelos examinados.

Analisado como variável categórica o OHIP-14 foi classificado em dois grupos: sem impacto (quase nunca e nunca) e com impacto (ocasionalmente, pouco e muito frequente), se o indivíduo declarou impacto em pelo menos um item do domínio foi pontuado impacto. ${ }^{18}$ As perguntas do índice foram agrupadas, duas a duas, e os domínios do instrumento foram estabelecidos:

1. Limitação funcional: Problemas de pronúncia (pergunta 1), paladar (pergunta 2). 
2. Dor física: dor (pergunta 3), incômodo na alimentação (pergunta 4).

3. Desconforto psicológico: desconforto com a condição bucal (pergunta 5), tensão nervosa (pergunta 6).

4. Incapacidade física: alimentação prejudicada (pergunta 7), interrupção de refeições (pergunta 8).

5. Incapacidade psicológica: dificuldade de relaxamento (pergunta 9), vergonha (pergunta 10).

6. Incapacidade social: irritação com outras pessoas (pergunta 11), dificuldade de fazer tarefas diárias (pergunta 12),

7. Desvantagem social: vida insatisfatória (pergunta 13) e incapacidade funcional (pergunta 14)

No levantamento das condições de saúde bucal foram utilizados o Índice de dentes cariados, perdidos e obturados (CPO-D), SiC Index e o Índice Periodontal Comunitário (IPC) de acordo com os critérios estabelecidos pela OMS para levantamento epidemiológico. ${ }^{19}$

$\mathrm{O}$ índice CPO-D foi utilizado para verificar a experiência de cárie dentária, ao passo que o SiC Index foi empregado para direcionar a atenção nos indivíduos com os valores mais altos de cárie. Este índice é calculado da seguinte forma: os indivíduos são ordenados de acordo com os valores CPO-D; um terço da população com os mais elevados índices de cárie são selecionados; a média CPO-D deste subgrupo é calculada. O resultado é o valor do $\mathrm{SiC}$ Index. ${ }^{19}$

Após a coleta dos dados, os questionários foram digitados em uma planilha eletrônica criada no software Epi Info, versão 3.5.1. Para a análise estatística, os oito níveis econômicos, previstos pela $\mathrm{ABEP}^{16}$, foram reagrupados da seguinte forma: A1, A2 e B1 em Nível Econômico Alto (A); B2, C1 e C2 em Nível Econômico Médio (M) e, D e E em Nível Econômico Baixo (B). Os sintomas do CPI também foram reagrupados para fins estatísticos sendo criados dois grupos: sem doença periodontal (sintoma ausente e sangramento) e com doença periodontal (presença de cálculo e bolsa periodontal). As respostas de auto-percepção apresentavam quatro possíveis respostas, sendo estas foram distribuídas na escala Likert e categorizadas em: Boa (excelente e boa) e Ruim (regular, ruim e péssima). Os testes estatísticos utilizados para verificação de associação entre as variáveis foram o teste Exato de Fisher com nível de significância de 5\% e análise de regressão logística.

A pesquisa foi aprovada pelo Comitê de Ética em Pesquisa com Seres Humanos (CEP) da Faculdade de Odontologia de Araçatuba - UNESP, segundo Resolução CNS 196 (Processo FOA-02492/2011).

\section{RESULTADOS}

Participaram da pesquisa 127 adolescentes grávidas. Quanto à idade, 94,5\% das se encontravam na adolescência tardia (16 a 19 anos) e 5,5\% na adolescência precoce (12 a 15 anos) na época do estudo. Não houve adolescentes nas classes sociais mais elevadas A1 e A2.

Do total, $59,1 \%$ eram solteiras; $58,2 \%$ moravam com seus familiares e $37,8 \%$ se declararam brancas (Tabela 1 ).

No que se refere à gravidez $92,9 \%$ das adolescentes afirmaram ser primigestas (primeiro filho). Das gestantes que já eram mães $88,9 \%$ afirmaram ter somente um filho (Tabela 1).

A grande maioria das adolescentes 63,0\% ( $\mathrm{n}=80)$ acreditavam ter algum problema em seus dentes e/ou gengivas e 17,3\% $(n=22)$ nunca foram ao cirurgião dentista. Das adolescentes que relataram ter acesso aos serviços odontológicos $60 \%$ afirmaram que o motivo da última consulta odontológica foi um problema ou necessidade (Tabela 1).

Tabela 1. Distribuição numérica e percentual de adolescentes grávidas, segundo as características sociodemográficas, fatores referentes à gestação, necessidade de tratamento, acesso aos serviços de saúde bucal e motivo da última consulta odontológica. Araçatuba-SP, Brasil, 2013

\begin{tabular}{|c|c|c|}
\hline Variável & $\mathbf{n}$ & $\%$ \\
\hline \multicolumn{3}{|l|}{ Nível Socioeconômico } \\
\hline Classe A1 & - & - \\
\hline Classe A2 & - & - \\
\hline Classe B1 & 14 & 11,0 \\
\hline Classe B2 & 34 & 26,8 \\
\hline Classe $\mathrm{C} 1$ & 40 & 31,5 \\
\hline Classe C2 & 28 & 22,0 \\
\hline Classe D & 10 & 7,90 \\
\hline Classe $\mathrm{E}$ & 01 & 0,80 \\
\hline \multicolumn{3}{|l|}{ Estado Civil } \\
\hline Solteiras & 76 & 59,8 \\
\hline Casadas & 50 & 39,7 \\
\hline Divorciadas & 01 & 0,80 \\
\hline \multicolumn{3}{|l|}{ Moradia } \\
\hline Familiares & 67 & 52,8 \\
\hline Companheiros & 49 & 38,6 \\
\hline Companheiros e filhos & 06 & 4,70 \\
\hline Sozinha & 05 & 3,90 \\
\hline \multicolumn{3}{|l|}{ Cor da Pele } \\
\hline Branca & 48 & 37,8 \\
\hline Parda & 41 & 32,3 \\
\hline Negra & 38 & 29,9 \\
\hline \multicolumn{3}{|l|}{ Primigesta } \\
\hline Sim & 117 & 92,1 \\
\hline Não & 010 & 7,90 \\
\hline \multicolumn{3}{|l|}{ Possui filhos } \\
\hline Sim & 009 & 7,10 \\
\hline Não & 118 & 92,9 \\
\hline Total & 127 & 100 \\
\hline \multicolumn{3}{|l|}{ Auto-percepção da saúde bucal } \\
\hline Boa & 52 & 41,0 \\
\hline Ruim & 75 & 59,0 \\
\hline $\begin{array}{l}\text { Acesso aos } \\
\text { odontológicos }\end{array}$ & & \\
\hline $\operatorname{Sim}$ & 105 & 82,7 \\
\hline Não & 22 & 17,3 \\
\hline Total & 127 & 100 \\
\hline \multicolumn{3}{|l|}{ Motivo da ultima consulta } \\
\hline \multirow{2}{*}{$\begin{array}{l}\text { Ocasional, tendo ou não algum } \\
\text { tipo de problema. } \\
\text { Problema ou necessidade. } \\
\text { Visita de rotina. }\end{array}$} & 36 & 34,3 \\
\hline & $\begin{array}{l}63 \\
06\end{array}$ & $\begin{array}{l}60,0 \\
5,70\end{array}$ \\
\hline Total & 105 & 100 \\
\hline
\end{tabular}

Observou-se uma variação individual de 0 a 45 pontos para o OHIP-14 (máximo possível: 56). Aproximadamente $21 \%$ das adolescentes entrevistadas responderam a todos os itens perguntados "nunca" (escore 0 ) relatando que possíveis problemas bucais não causaram impacto na sua qualidade de vida.

No Quadro 1 estão apresentadas as distribuições de frequências das respostas, segundo cada item do OHIP-14. Os itens que demonstraram maior impacto foram a dor e o incômodo com a alimentação sentidos devido a problemas bucais, manifestados por $23,6 \%$ e $18,9 \%$ das participantes. Sendo que os itens que apresentaram maior frequência de respostas em que não se detectaram impacto foram os que compõem o sexto e sétimo domínio (itens 12 a 14).

Os domínios, que representaram maior impacto foram o domínio "dor física", relativo às perguntas "Você sentiu dor em sua boca, seus dentes ou dentadura?" (item 3) e "Sentiu-se incomodado ao comer algum alimento por causa de sua boca, seus dentes ou dentadura?" (item 4) apontados por $23,7 \%$ das adolescentes, seguido do domínio 
"incapacidade física", relativo as perguntas "Sua alimentação ficou prejudicada por causa de sua boca, seus dentes ou dentadura?"(item 7) e "Você teve que parar suas refeições por causa de problemas em sua boca, seus dentes ou dentadura?" (item 8) apontados por $19,7 \%$ das adolescentes (Tabela 2).

Quadro 1- Estatística descritiva do impacto relatado para cada item do OHIP-14, entre adolescentes grávidas, apresentando o total percentual de cada ponto da escala (0 a 4). Araçatuba-SP, Brasil, 2013.

\begin{tabular}{|c|c|c|c|c|c|c|c|c|c|c|}
\hline \multirow{3}{*}{ Impactos Investigados } & \multicolumn{10}{|c|}{ Frequência } \\
\hline & \multicolumn{2}{|c|}{$\begin{array}{c}0 \\
\text { (nunca) }\end{array}$} & \multicolumn{2}{|c|}{$\begin{array}{c}1 \\
\text { (quase nunca) }\end{array}$} & \multicolumn{2}{|c|}{$\begin{array}{c}2 \\
\text { (ocasionalmente) }\end{array}$} & \multicolumn{2}{|c|}{$\begin{array}{c}3 \\
\text { (pouco freqüente) }\end{array}$} & \multicolumn{2}{|c|}{$\begin{array}{c}4 \\
\text { (muito } \\
\text { freqüente) }\end{array}$} \\
\hline & $\mathrm{n}$ & $\%$ & $\mathrm{n}$ & $\%$ & $\mathrm{n}$ & $\%$ & $\mathrm{n}$ & $\%$ & $\mathrm{n}$ & $\%$ \\
\hline $\begin{array}{l}\text { 1. Teve problemas para falar alguma } \\
\text { palavra }\end{array}$ & 85 & 66,9 & 26 & 20,5 & 10 & 7,9 & 6 & 4,7 & - & - \\
\hline $\begin{array}{l}\text { 2.Sentiu que o sabor dos alimentos } \\
\text { mudou }\end{array}$ & 70 & 55,1 & 36 & 28,3 & 16 & 12,6 & 4 & 3,1 & 1 & 0,8 \\
\hline 3.Sentiu dores em sua boca e dentes & 56 & $\overline{44,1}$ & 41 & 32,3 & 18 & 14,2 & 7 & 5,5 & 5 & 3,9 \\
\hline 4. Sentiu-se incomodado ao comer & 62 & 48,8 & 41 & 32,3 & 15 & 11,8 & 6 & 4,7 & 3 & 2,4 \\
\hline 5. Você ficou preocupada & 72 & 56,7 & 36 & 28,3 & 14 & 11,0 & 3 & 2,4 & 2 & 1,6 \\
\hline 6. Sentiu-se estressada & 73 & 57,5 & 34 & 26,8 & 14 & 11,0 & 5 & 3,9 & 1 & 0,8 \\
\hline 7. Sua alimentação ficou prejudicada & 73 & 57,5 & 29 & 22,9 & 14 & 11,0 & 7 & 5,5 & 4 & 3,1 \\
\hline 8. Você teve que parar suas refeições & 72 & 56,7 & 38 & 29,9 & 5 & 7,9 & 5 & 3,9 & 2 & 1,6 \\
\hline 9. Encontrou dificuldade para relaxar & 71 & 55,9 & 34 & 26,8 & 18 & 14,2 & 3 & 2,4 & 1 & 0,8 \\
\hline 10. Sentiu-se envergonhada & 66 & 52 & 40 & 31,5 & 16 & 12,6 & 3 & 2,4 & 2 & 1,6 \\
\hline 11. Irritação com pessoas & 78 & 61,4 & 35 & 27,6 & 11 & 8,7 & 2 & 1,6 & 1 & 0,8 \\
\hline $\begin{array}{l}\text { 12. Dificuldade na realização de } \\
\text { atividades diárias }\end{array}$ & 84 & 66,1 & 32 & 25,2 & 8 & 6,3 & 2 & 1,6 & 1 & 0,8 \\
\hline 13. Sentiu a vida piorar & 86 & 67,7 & 28 & 22,0 & 10 & 7,9 & 1 & 0,8 & 2 & 1,6 \\
\hline $\begin{array}{l}\text { 14. Total incapacidade na realização das } \\
\text { atividades diárias }\end{array}$ & 99 & 77,9 & 19 & 15,0 & 8 & 6,3 & - & - & 1 & 0,8 \\
\hline
\end{tabular}

Tabela 2. Distribuição de frequência do impacto do OHIP-14 em adolescentes grávidas categorizado por domínio. Araçatuba-SP, Brasil, 2013

\begin{tabular}{|c|c|c|c|c|}
\hline \multirow{2}{*}{ Domínio } & \multicolumn{2}{|c|}{ Sem impacto } & \multicolumn{2}{|c|}{ Com Impacto } \\
\hline & $\mathbf{n}$ & $\%$ & $\mathbf{n}$ & $\%$ \\
\hline Limitação funcional & 106 & 87,6 & 21 & 12,4 \\
\hline Dor física & 97 & 76,3 & 30 & 23,7 \\
\hline $\begin{array}{l}\text { Desconforto } \\
\text { psicológico }\end{array}$ & 107 & 84,2 & 20 & 15,8 \\
\hline Incapacidade física & 102 & 80,3 & 25 & 19,7 \\
\hline $\begin{array}{l}\text { Incapacidade } \\
\text { psicológica }\end{array}$ & 106 & 83,4 & 21 & 16,6 \\
\hline Incapacidade social & 113 & 88,9 & 14 & 11,1 \\
\hline Desvantagem social & 114 & 89,7 & 13 & 10,3 \\
\hline
\end{tabular}

A prevalência da cárie entre as adolescentes foi de 93,7\% ( $\mathrm{n}=119)$, e a média do índice CPO-D de 12,54 com desvio padrão de 4,26 (Tabela 3). Já o valor do SiC Index encontrado foi de 18,85 .

Tabela 3. Estatística descritiva dos componentes cariados, perdidos, obturados, do índice CPO-D de adolescentes grávidas.

$$
\text { Araçatuba-SP, Brasil, } 2013
$$

\begin{tabular}{lcccc}
\hline & Cariado & Perdido & Obturado & CPO-D \\
\hline Média & 8,87 & 0,12 & 3,55 & 12,54 \\
Desvio & 4,69 & 0,51 & 2,54 & 4,26 \\
padrão & 0,00 & 0,00 & 0,00 & 0,00 \\
Mínimo & 6,00 & 0,00 & 1,50 & 10,00 \\
$1^{\circ}$ Quartil & 9,00 & 0,00 & 4,00 & 13,00 \\
Mediana & 12,00 & 0,00 & 5,00 & 16,00 \\
$3^{\circ}$ Quartil & 19,00 & 4,00 & 14,00 & 20,00 \\
Máxima & & & & \\
\hline
\end{tabular}

Quanto ao IPC observou-se que $92,1 \%$ das adolescentes apresentavam algum tipo de comprometimento periodontal, sendo que a presença de sangramento gengival foi o sintoma mais frequente entre os sextantes examinados (Tabela 4).

Tabela 4- Valor do Índice Periodontal Comunitário em adolescentes Grávidas. Araçatuba-SP, Brasil, 2013

\begin{tabular}{ccccccc}
\hline \multirow{2}{*}{ Condição } & $\mathbf{1 6 / 1 7}$ & $\mathbf{1 1}$ & $\mathbf{2 6 / 2 7}$ & $\mathbf{3 6 / 3 7}$ & $\mathbf{3 1}$ & $\mathbf{4 6 / 4 7}$ \\
\hline Excluído & 00,0 & 00,0 & 00,0 & 00,0 & 00,0 & 00,0 \\
Hígido & 22,1 & 69,3 & 25,2 & 18,9 & 59,6 & 18,6 \\
Sangramento & 52,7 & 26,8 & 45,7 & 47,3 & 35,3 & 51,1 \\
Cálculo & 20,4 & 02,4 & 22,9 & 20,5 & 03,0 & 20,2 \\
$\begin{array}{c}\text { Presença de bolsa } \\
\text { superficial/ profunda }\end{array}$ & 04,8 & 01,5 & 06,2 & 13,3 & 02,1 & 10,1 \\
\hline
\end{tabular}

A Tabela 5 apresenta os resultados dos testes de associação entras as variáveis do estudo.

$\mathrm{Na}$ análise de regressão logística as variáveis analisadas foram: impacto na saúde bucal, adolescência tardia ou precoce (idade), necessidade de tratamento percebida, saúde bucal, autopercepção da saúde bucal, livre de cárie e acesso aos serviços odontológicos. Onde se verificou associação estatisticamente significante entre o impacto da saúde bucal na qualidade de vida e autopercepção em saúde bucal (Tabela 6). 
Tabela 5. Associação entre as variáveis: impacto na qualidade de vida, saúde bucal, condição socioeconômica, necessidade e acesso ao tratamento odontológico. Araçatuba - SP, Brasil, 2013

\begin{tabular}{|c|c|c|c|}
\hline $\begin{array}{c}\text { Variáveis } \\
\text { Impacto na qualidade de vida } \\
\mathrm{X} \\
\text { Doença periodontal }\end{array}$ & Teste de Associação & $\begin{array}{l}\text { p-valor } \\
0,0311\end{array}$ & Significância* \\
\hline $\begin{array}{c}\text { Impacto na qualidade de vida } \\
\mathrm{X} \\
\text { Necessidade de tratamento }\end{array}$ & Teste Exato de Fisher & 0.3179 & NS \\
\hline $\begin{array}{c}\text { Impacto na qualidade de vida } \\
\text { X } \\
\text { Nível socioeconômico }\end{array}$ & Teste Exato de Fisher & 0.4867 & NS \\
\hline $\begin{array}{l}\text { Doença periodontal } \\
\text { X } \\
\text { Nível socioeconômico }\end{array}$ & Teste Exato de Fisher & 0.4479 & NS \\
\hline $\begin{array}{c}\text { Autopercepção } \\
\text { X } \\
\text { Doença periodontal }\end{array}$ & Teste Exato de Fisher & 0,0166 & $\mathrm{~S}$ \\
\hline
\end{tabular}

* NS= Não significante S Significante

Tabela 6. Modelo final de regressão logística para o impacto dos problemas de saúde bucal sobre a qualidade de vida (OHIP - prevalência) considerando "o impacto dos problemas bucais" como variável resposta. Araçatuba - SP, Brasil, 2013

\begin{tabular}{|c|c|c|c|c|c|c|c|}
\hline \multirow[b]{2}{*}{ Parameter } & \multicolumn{6}{|c|}{ Analysis of Maximum Likelihood Estimates } & \multirow[b]{2}{*}{$\begin{array}{c}95 \% \text { Wald } \\
\text { Confidence Limits }\end{array}$} \\
\hline & DF & Estimate $(\beta)$ & $\begin{array}{r}\text { Standard } \\
\text { Error }\end{array}$ & $\begin{array}{r}\text { Wald } \\
\text { Chi-Square }\end{array}$ & Pr $>$ ChiSq & OR & \\
\hline Intercept & 1 & -1.9459 & 0.3381 & 33.1324 & $<0001$ & & - \\
\hline Autopercepção & 1 & 1.2845 & 0.4572 & 7.8929 & 0.0050 & 3.613 & $1.475-8.852$ \\
\hline
\end{tabular}

\section{DISCUSSÃO}

Nessa pesquisa foi observado que a maioria das participantes relatou impacto na qualidade de vida em decorrência das condições bucais. Em estudos semelhantes da área da ortodontia conduzidos com adolescentes, verificou-se também alta prevalência de impacto de questões bucais na qualidade de vida demonstrando que os problemas bucais podem ser grandes influenciadores de impacto negativo na qualidade de vida tanto por questões estéticas como funcionais ${ }^{20,21}$.

O baixo escore de OHIP médio encontrado, também verificado no estudo de Biazevic et. al. ${ }^{11}$, sugere que o impacto relatado na qualidade de vida em decorrência das condições bucais mesmo quando presente parece ser de pequena intensidade. Os domínios que mais contribuíram para o escore total do OHIP foram "dor física" e "incomodo com a alimentação", com prevalência de impacto de 55,9\% e $51,2 \%$ entra as adolescentes. Fato que também pode ser observado em estudos de Biazevic et al. ${ }^{11}$ e Onyeaso ${ }^{22}$ nos quais a "dor física" também foi apontada como o item do OHIP mais frequentemente citado.

Nesse estudo sobre gestação e saúde bucal na adolescência observou-se que a maior parte das gestantes estava na adolescência tardia (mais de 15 anos) o que corrobora com os dados do Ministério da Saúde ${ }^{23}$, que indicou que em meninas com idade inferior a 15 anos (fase precoce da adolescência), a taxa de gestação é de $0,9 \%$ e para aquelas entre os 15 e os 19 anos (fase tardia da adolescência) é de $22,6 \%$.

Os dados obtidos referentes aos fatores socioeconômicos delineiam um perfil de adolescentes pertencentes às classes mais baixas, o que pode ser explicado pelo fato de que a população alvo desse estudo eram usuárias de um sistema público de saúde, 59,1\% das adolescentes eram solteiras, resultado abaixo do encontrado pela Pesquisa Nacional de Demografia e Saúde da Criança e da Mulher- PNDS- 2006, pesquisa de grande representatividade nacional ${ }^{24}$, que denotou $72,1 \%$ mulheres solteiras entre 15 e 19 anos. Já em relação à moradia mais de $50 \%$ viviam com os pais, padrão que se repete em capitais brasileiras $^{25}$.

Apenas 7,1\% das adolescentes já eram mães, dados que diferem do estudo realizado pelo "Centers for Disease Control and Prevention (CDC)" dos Estados Unidos, que encontrou taxas de 19,5\% em 2007 e 18,3\% em $2010 .{ }^{26}$ Essa diferença pode ser explicada pelo fato de que todas as adolescentes que participaram da pesquisa estavam em acompanhamento pré-natal. Pesquisas entre mães adolescentes tem demosntrado que o aconselhamento prénatal associado com o aumento da probabilidade de utilização de métodos de eficazes, está associado com taxas reduzidas de repetição da gravidez entre as adolescentes $^{21,27,28}$

Ao analisar as variáveis: impacto na saúde bucal e saúde bucal, foi encontrada uma diferença estatisticamente significante $(p=0,0311)$. Já análise multivariada mostrou que essa variável está associada à autopercepção da saúde bucal sobre a qualidade de vida. $\mathrm{O}$ que pode ser explicado pelo fato de que a amostra estudada foi $100 \%$ do sexo feminino, acredita-se que mulheres são mais críticas em relação a própria saúde. Estudos como de Slade e Spencer ${ }^{12}$ afirmam 
que mulheres têm 2,08 vezes mais chances de terem impacto na qualidade de vida do que indivíduos do sexo masculino.

No que se refere à condição de saúde bucal, o CPOD médio de 12,51 entre as gestantes examinadas é considerado alto, mas foi semelhante ao encontrado por Zanata et al. ${ }^{29}$ em 2003. Por outro lado, foi maior do que observado em estudos de Ramos et al. ${ }^{30}$ (CPOD=10,4), de Tonello et al.$^{31}$ $(\mathrm{CPOD}=11,08)$ e de Villa et al. ${ }^{32}(\mathrm{CPOD}=8,0)$.

Uma análise mais detalhada da situação da cárie em muitos países mostra que há uma distribuição assimétrica da prevalência de cárie, pois uma proporção da população ainda tem valores de CPO-D altos, enquanto outra proporção é totalmente livre de cárie. A média do CPO-D não reflete distribuições assimétricas, podendo levar à conclusão incorreta de que a situação da cárie para toda a população está controlada, quando, na realidade, muitos indivíduos ainda têm cáries ${ }^{33}$.

Portanto, a fim de observar a polarização da cárie dentária, foi empregado o $\mathrm{SiC}$ Index, e encontrou-se um valor médio de 18, 85 no grupo em questão. Comparando-se estes resultados com os de um estudo realizado na Dinamarca $^{34}$ em 1993, apenas 9,0\% dos jovens entre 18 e 25 anos tinham CPO-D $\geq 12,0$; portanto, apesar de Araçatuba estar localizada no Estado de São Paulo o mais populoso e industrializado do país, a prevalência de cárie permanece alta entre as adolescentes grávidas, demonstrando a necessidade de programas em saúde bucal que atendam às reais necessidades dessa população.

O SiC Index pode, também, trazer importantes informações adicionais sobre o impacto da doença nos indivíduos mais afetados. Marthaler ${ }^{35}$ reforça a importância dessa ferramenta, pois, por ser uma média, não depende de avaliações do nível socioeconômico e, consequentemente, das possíveis formas de interpretação que podem ocorrer de um país para outro, permitindo, assim, comparações entre populações distintas. Cabe ressaltar também, que a gestação na adolescência não pode ser considerada fator determinante para a doença cárie, mas as condições biológicas e psicossociais em que a maioria das adolescentes se encontra nessa fase, assim como o limitado conhecimento sobre as técnicas de higiene bucal que podem levar ao desenvolvimento de novas lesões de cárie ou agravar aquelas pré-existentes.

Já o sangramento gengival observado em mais da metade $(85,0 \%)$ das gestantes adolescentes pode sugerir uma tendência ao sangramento durante a gestação, uma vez que o aumento nos níveis circulantes de progesterona causa dilatação dos capilares gengivais, permeabilidade e liberação de exudato gengival, o que pode explicar essa tendência à vermelhidão e ao aumento no sangramento durante o período gestacional ${ }^{36}$.

A condição periodontal brasileira avaliada pelo Índice Periodontal Comunitário (IPC) segundo o Manual SB Brasil 2010 apontou, 50,9\% de doença periodontal entre 15 e 19 $\operatorname{anos}^{37}$, valor abaixo do que foi encontrado em nosso estudo. Entretanto a prevalência de doença periodontal observada nas puérperas do presente estudo foi semelhante à relatada por Santana et al. ${ }^{38}$ que detectaram $91,2 \%$ de doença periodontal entre as gestantes de uma maternidade pública do município de Pernambuco e Moimaz et al. ${ }^{39}$ que relataram $92 \%$ de doença periodontal em unidades de saúde de dois municípios paulistas.

Sabe-se que a doença periodontal e a cárie dentária são doenças acumulativas, que podem ocasionar dor, limitação funcional, comprometimentos estéticos e preocupação ou desapontamento em relação à boca ou dentes, comprometendo vários aspectos da qualidade de vida relacionada à saúde bucal. Entretanto não houve associação significante a condição de saúde bucal e OHIP-14 em nosso estudo.

Uma vez que a qualidade de vida está relacionada às expectativas, padrões e preocupações do indivíduo, este estudo sugere que adolescentes grávidas têm diminuição em sua autoestima o que pode piorar os resultados em questões relacionadas à autopercepção. Assim, condições que podem interferir em aspectos biopsicossociais, tais, como a saúde bucal, tendem ater mais relevância para esses indivíduos podendo ocasionar maior impacto na qualidade de vida dos mesmos.

\section{CONCLUSÃO}

Por se tratar de um estudo realizado em Unidades Básicas de Saúde não houve gestantes nos níveis sociais mais elevados. As adolescentes apresentaram alto índice de cárie dentária e doença periodontal, revelando saúde bucal deficiente. A maioria relatou impacto na qualidade de vida em decorrência de problemas bucais, sendo a presença de "dor física" a maior responsável pelas repercussões negativas na vida destas meninas.

\section{REFERÊNCIAS}

1. Organização Mundial da Saúde (1965). Problemas de la salud de La adolescencia. Informe de un comité de expertos de la O.M.S (Informe técnico $\mathrm{n}^{\circ}$ 308). Genebra.

2. Brêtas JRS, Ohara CVS, Jardim DP, Aguiar Junior W, Oliveira JR. Aspectos da sexualidade na adolescência. Ciênc saúde. 2011;16(7): 3221-8.

3. Instituto Brasileiro de Geografia e Estatística (IBGE). Síntese de indicadores sociais. Rio de Janeiro: IBGE; 2010.

4. Ferreira MA, Alvim NAT, Teixeira MLO, Veloso RC Saberes de adolescentes: estilo de vida e cuidado à saúde. Texto Contexto Enferm. 2007;16(2):217-24.

5. Källestål C, Dahlgren L, Stenlund H. Oral health behavior and self-esteem in Swedish adolescents over four years. J Adolesc Health. 2006; 38(5):583-90.

6. Garbin CAS, Sumida DH, Santos RR, Chehoud KA, Moimaz SAS. Oral health promotion during pregnancy. Rev Odontol UNESP. 2011; 40(4):161-5.

7. Antunes JLF, Peres MA, Frias AC, Crosato EM, Biazevic MGH. Saúde gengival de adolescentes e a utilização de serviços odontológicos, Estado de São Paulo. Rev Saúde Pública. 2008; 42(2):191-9.

8. Petersen PE, Bourgeois D, Ogawa H, Estupinan-Day S, Ndiaye C. The global burden of oral diseases and risks to oral health. Bull World Health Organ. 2005; 83(9):661-9.

9. Clark WB, Löe H. Mechanisms of initiation and progression of periodontal disease. Periodontol 2000 1993; 2: 72-82.

10. Moimaz SAS, Rocha NB, Saliba O, Garbin CAS. O acesso de gestantes ao tratamento odontológico. Rev Odontol Univ Cidade São Paulo. 2007; 19(1):39-45.

11. Biazevic MGH, Araújo ME, Michel-Crosato E. Indicadores de qualidade de vida relacionados com a saúde bucal: revisão sistemática. UFES Rev Odontol. 2002; 4(2):13-25.

12. Slade GD, Spencer AJ. Development and evaluation of the oral health impact profile. Community Dent Health. 1994; 11(1):3-11. 
13. Locker D. Measuring oral health: a conceptual framework. Community Dental Health. 1988; 5(1):5-13.

14. Sanders AE, Slade GD, Lim S, Reisine, ST. Impact of oral disease on quality of life in the US and Australian populations. Community Dent Health. 2009; 37(2):17181.

15. Slade GD, Hoskin GW, Spencer AJ. Trends and flutuations in the impact of oral conditions among older adults during a one year period. Commun Dent Oral Epidemiol. 1996;24(5):317-21.

16. Associação Brasileira de Empresas de Pesquisa -ABEPCritério Padrão de Classificação Econômica Brasil/2008. [citado 2013 abril 05]. Disponível em: http://www.viverbem.fmb.unesp.br/docs/classificacaobr asil.pdf

17. Slade GD. Derivation and validation of a short-form oral health impact profile. Community Dent Oral Epidemiol. 1997; 25(4):284-90.

18. World Health Organization. Oral health surveys: basic methods. $3^{\text {rd }}$ ed. Geneva: ORH/EPID; 1987.

19. Word Health Organization. Significant Caries Index SiC. http://www.mah.se/CAPP/Methods-andIndices/for-Caries-prevalence/ (acessado em 15/set/2013).

20. Tocce KM, Sheeder JL, Teal SB. Rapid repeat pregnancy in adolescents: do immediate postpartum contraceptive implants make a difference? Am J Obstet Gynecol. 2012; 206(6):481.e1-7.

21. De Paula Júnior DF, Santos NC, da Silva ET, Nunes MF, Leles CR. Psychosocial impact of dental esthetics on quality of life in adolescents. Angle Orthod. 2009;79(6):1188-93.

22. Onyeaso CO. Orthodontic treatment complexity and need with associated oral health related quality of life in Nigerian adolescents. Oral Health Prev Dent. 2009;7(3):235-41.

23. Ministério da Saúde. Secretaria de Atenção à Saúde. Departamento de ações Programáticas Estratégicas. PréNatal e Puerpério: atenção qualificada e humanizada. Brasília. Ministério da Saúde 2006.

24. Ministério da Saúde. PNDS 2006 - Pesquisa Nacional de Demografia e Saúde da Criança e da Mulher. Brasília: MS; 2008

25. Vonk ACRP, Bonan C, Silva KS. Sexualidade, reprodução e saúde: experiências de adolescentes que vivem em município do interior de pequeno porte. Ciênc saúde coletiva, 2013;18(3):1795-1807.

26. Centers for Disease Control and Prevention (CDC). Vital signs: Repeat births among teens - United States, 2007-2010. MMWR Morb Mortal Wkly Rep. 2013;62(13):249-55.

27. Wilson EK, Fowler CI, Koo HP. Postpartum contraceptive use among mothers in seven states. J Adolesc Health 2012;52(3):278-83.

28. Tocce KM, Sheeder JL, Teal SB. Rapid repeat pregnancy in adolescents: do immediate postpartum contraceptive implants make a difference? Am J Obstet Gynecol. 2012;206(6):481.e1-7.

29. Zanata RL, Na varro MF , Pereira JC, Franco EB , Lauris JR, Barbosa SH. Effect of caries preventive measures directed to expectant mothers on caries experience in their children. Braz Dent J. 2003; 14(2):75-81.

30. Ramos TM, Almeida Junior AA, Ramos TM, Novais SM A, Grinfeld S, Fortes TMV, Pereira MAS Condições bucais e hábitos de higiene oral de gestantes de baixo nível sócio-econômico no município de Aracaju-SE. Pesq Bras Odontoped Clin Integr. 2006;6(3):229-235.

31. Tonello AS, Zuchieri MABO, Pardi V. Assessment oforal health status of pregnant women participating in a family health program in the city of Lucas do Rio Verde-MT-Brazil. Braz J Oral Sci. 2007; 6(20):1265-8.

32. Villa A, Abati S, Pileri P, Calabrese S, Capobianco G, Strohmenger $\mathrm{L}$, et al. Oral health and oral diseases in pregnancy: a multicentre survey of Italian postpartum women. Aust Dent J. 2013;58(2):224-9

33. Pitts NB, Evans DJ, Nugent ZJ, Pine CM. The dental caries experience of 12-year-old children in England and Wales. Surveys coordinated by the British Association for the Study of Community Dentistry in 2000/2001. Community Dent Health. 2002; 19(1):46-53.

34. Antoft P, Rambusch E, Antoft B, Christensen HW. Caries experience, dental health behaviour and social status - Three comparative surveys among Danish military recruits in 1972, 1982 and 1993. Community Dent Health. 1999; 16(2):80-4.

35. Marthaler TM. Changes in dental caries 1953- 2003. Caries Res 2004; 38:173-81.

36. Dhaliwal JS, Lehl G, Sodhi SK, Sachdeva S. Evaluation of socio-demographic variables affecting the periodontal health of pregnant women in Chandigarh, India. J Indian Soc Periodontol. 2013;17(1):52-7.

37. Mistério da Saúde. Secretaria de Atenção a Saúde. Departamento de Atenção Básica. Projeto SBBrasil 2010: Pesquisa Nacional de Saúde Bucal -Resultados Principais. Brasília: Ministério da Saúde, 2011.

38. Santana FCM, Santos VIM, Freire S, Couto GBL. The periodontal disease as a risk factor in preagnants with premature childbird and low birth weight. Pesqui Bras Odontopediatria Clín Integr. 2005;5(3):247-52.

39. Moimaz SAS, Garbin CAS, Zina LG, Carmo MP, Saliba NA. Periodontite materna e nascimento de bebes prétermo ou de baixo peso - existe associação? Cienc Odontol Bras. 2009;12(2):61-9.

\section{CONFLITO DE INTERESSES}

Os autores declaram não haver conflitos de interesse.

\section{AUTOR PARA CORRESPONDÊNCIA}

\section{Daniela Pereira Lima}

dani.pl@hotmail.com

Submetido em 09/09/2016 Aceito em 19/09/2016 